\title{
Selection for increased quorum-sensing cooperation in Pseudomonas aeruginosa through the shut-down of a drug resistance pump
}

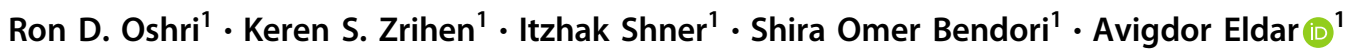

Received: 22 January 2018 / Revised: 10 May 2018 / Accepted: 15 May 2018 / Published online: 20 June 2018

(c) The Author(s) 2018. This article is published with open access

\begin{abstract}
The opportunistic pathogen Pseudomonas aeruginosa employs a hierarchical quorum-sensing network to regulate virulence factor production that cooperatively benefit the population at a cost to the individual. It has been argued that the evolution of a cooperative mutant in a quorum sensing-suppressed population would be hampered through its exploitation by neighboring non-mutant cells. It remains unclear whether mechanisms which overcome this exploitation exist. Here we investigate the regain of quorum-sensing cooperation by evolving a mutant of the las $R$ master quorum-sensing regulator. The mutant regained partial cooperative growth through null mutations in mexT, which codes for an activator of the MexEF-OprN multidrug-resistant pump. We find that these mutations enhance cooperative growth in both the las $R$ mutant and wild-type backgrounds through the activation of the RhIIR system. We show that the regain of cooperation in mexT mutants is mediated by the reduction in MexEF-OprN activity, whereas an additional source of private benefit is mostly mexEF-oprNindependent. Finally, we show that addition of antibiotics for which resistance is mediated by MexEF-OprN prevents the selection of increased cooperation at sub-MIC concentrations. MexT, therefore, not only links private and public goods, but also exposes conflicts between selection for antibiotic resistance and enhanced cooperation.
\end{abstract}

\section{Introduction}

Bacteria engage in altruistic cooperation by secreting a variety of costly molecules which modify the environment to the benefit of the bacterial community. Such public goods molecules include enzymes, toxins, antibiotics, and surfactants [1-3]. Public goods secretion is often regulated by quorum-sensing cell-cell signaling, which depends on the release of a signal molecule and its detection by a cognate receptor [4, 5]. Quorum sensing leads to a densitydependent response, which improves the efficiency of public goods utilization [6-8].

These authors contributed equally: Ron D. Oshri, Keren S. Zrihen

Electronic supplementary material The online version of this article (https://doi.org/10.1038/s41396-018-0205-y) contains supplementary material, which is available to authorized users.

Avigdor Eldar avigdor@gmail.com

1 School of Molecular Cell Biology and Biotechnology, Faculty of Life Sciences, Tel-Aviv University, Tel-Aviv, Israel
Regulation of secretion by quorum sensing is crucial for the lifestyle of the opportunistic pathogen Pseudomonas aeruginosa. This species has a complex network of quorumsensing systems, consisting of the LasIR and RhlIR systems whose signal molecules belong to the Acyl-homoserinelactone family and the Pseudomonas quinolone signal (PQS) system, which codes for several quinolone signal molecules [9]. These systems are organized hierarchically with the Las system at the top tier, regulating the activation of the Rhl and PQS systems, which also cross-regulate in a complex manner [10-13]. The quorum-sensing network of $P$. aeruginosa controls the secretion of a variety of virulence factors including all secreted proteases $[12,14,15]$.

An intrinsic problem with quorum sensing-dependent cooperative secretion is its susceptibility to exploitation by quorum-sensing mutants, which do not secrete the public goods, but enjoy their benefits [5]. Such cheater mutants gain an increased fitness over the wild-type strain and are therefore able to invade the population and reduce the level of cooperation or even lead to a population collapse [16, 17]. Indeed, las $R$ mutants of $P$. aeruginosa have been shown to evolve when the wild-type bacteria were grown in a medium whose only carbon source is casein, which 
requires quorum-sensing-dependent secretion of proteases for its digestion [18, 19]. Interestingly, las $R$ mutants also emerge during chronic infections, though the question of whether they arise by cheating is yet unresolved [20-22].

A related question is whether cooperative variants would be able to invade a community dominated by cheater genotypes. This general question has been previously explored in the fruiting body formation process of Myxococcus xanthus [23, 24], yeast secretion of invertase [25] and $P$. aeruginosa siderophore secretion [26]. A similar problem arises in the context of evolution of resistance to antiquorum-sensing drugs, where a resistant mutant will be counter-selected thorough exploitation by its non-mutated neighbors $[5,27,28]$. This theoretical claim was demonstrated in a simple setting for $P$. aeruginosa, by reversing the role of a lasR mutant (which represented the inhibited wild-type) and the wild-type (which represented the inhibitor-resistant mutant) [29, 30]. These experiments do not rule out the evolution of other compensatory mutations, or the evolution of resistance in structured population [31, 32].

In the case of $P$. aeruginosa, the effect of a las $R$ deletion (or LasR inhibition by drugs) on public goods secretion may be partially complemented by a mutation resulting in a Las-independent activation of the Rhl system. Decoupling of the Las and Rhl systems has been demonstrated to occur under certain environmental conditions [10, 33-35]. Therefore, mutations that will extend the range of conditions in which decoupling occurs, are likely to emerge. As in other cases, it is unclear whether such mutant will be able to overcome exploitation by neighboring ancestral cells. Interestingly, recent analysis of clinically evolved las $R$ mutant isolates, indicates that some have partially reactivated their Rhl system [20].

Two decades ago, the Iglewski group evolved a las $R$ mutant in casein medium. They obtained a suppressive mutation that led to overproduction of the Rhl signal molecule and allowed the cells to regain their cooperative growth [36]. Here, we revisit this experiment with modern sequencing tools to identify the causal mutations that shape the regain of cooperation. We show that this regain can be attributed to an inactivation of the mexT transcriptional regulator, which activates the expression of the multidrugresistant pump MexEF-OprN. This regulatory mutation has two main contributions to fitness; it leads to a regain of cooperation through induction of the Rhl system via a MexEF-OprN-dependent mechanism, and privately benefit the cells via a mostly MexEF-OprN-independent mechanism. As the MexEF-OprN pump is crucial for antibiotic resistance, this mechanism leads to a conflict between the evolutionary regain of cooperation and antibiotic resistance.

\section{Materials and methods}

\section{Bacterial strains and plasmids}

Bacterial strains used in this study are listed in Supplementary Tables S1, S2. Strains were either acquired from other laboratories (Greenberg, Banin, Köhler) or arose by evolution. The $\operatorname{mexT} ; \Delta l a s R$ deletion strain was constructed by introducing the las $R$ deletion into strain AEA325 as previously described [37]. All promoter reporter plasmids were cloned into the pPROBE plasmid (kindly provided by the Banin laboratory) [38], using the primers described in Supplementary Table S3 with standard cloning methods. Constitutive BFP and GFP reporter plasmids were constructed using the backbone of pMRP9-1 [39], under the control of the constitutive $\mathrm{P}_{\mathrm{A1} / 04 / 03}$ promoter [40]. Both BFP and GFP were synthetically designed as $P$. aeruginosa codon-optimized variants and obtained from GENEWIZ (Plainfield, USA). The amino-acid sequences of GFP and BFP are of GFPmut2, and Azurite, respectively.

\section{Growth conditions}

Routine growth of cultures was done in lysogeny broth (LB) medium. Antibiotic was added when needed, at the following concentrations, Escherichia coli, $100 \mu \mathrm{g} / \mathrm{mL}$ ampicillin; P. aeruginosa, $300 \mu \mathrm{g} / \mathrm{mL}$ carbenicillin. For the relevant experiments (Fig. 4), chloramphenicol (Cm) stock at a concentration of $50 \mu \mathrm{g} / \mathrm{mL}$ in ethanol was diluted into the various final concentrations. We found that ethanol $(\mathrm{Cm}$ carrier) levels had a significant effect on growth in casein and therefore carrier levels were kept constant when comparing growth at different $\mathrm{Cm}$ concentrations. CasaminoAcids (CAA) and casein media were made as previously reported [18]. 0.5\% CAA medium: M8 minimal medium (5 $\mathrm{g} / \mathrm{L} \mathrm{Na} \mathrm{HPO}_{4} * 2 \mathrm{H}_{2} \mathrm{O}, 3 \mathrm{~g} / \mathrm{L} \mathrm{K \textrm {K } _ { 2 }} \mathrm{PO}_{4}, 0.5 \mathrm{~g} / \mathrm{L} \mathrm{NaCl}, 1 \mathrm{mM}$ $\mathrm{MgSO}_{4}, 100 \mu \mathrm{M} \mathrm{CaCl}_{2}$ ) and $5 \mathrm{~g} / \mathrm{L}$ Acid-hydrolyzed casein (Difco), adjusted to $\mathrm{pH}=7.4 .1 \%$ casein medium: M8 minimal medium and $10 \mathrm{~g} / \mathrm{L}$ casein sodium salt from bovine milk (Difco), adjusted to $\mathrm{pH}=7.4$.

\section{Evolution of $\Delta$ las $R$ in casein}

Fresh colonies of $P$. aeruginosa PAO1 $\Delta$ lasR strain (AEA102) were inoculated into $2 \mathrm{~mL}$ of $0.5 \%$ CAA medium and were cultured for $16 \mathrm{~h}$ at $37^{\circ} \mathrm{C}$ with agitation. The following morning, $\mathrm{OD}_{600}$ of cultures was usually $1-2$. The cultures were diluted to an $\mathrm{OD}_{600}$ of 0.1 into $1 \%$ casein medium and were left at $37{ }^{\circ} \mathrm{C}$ with agitation. Significant opacity was observed 32-37 days post incubation. Cells were incubated for 3 more days after the increase in opacity prior to isolation of mutants. 


\section{Evolution of wild-type PA01 $\left(\right.$ mexT $\left.^{+}\right)$in casein}

Initial culturing was performed as above. Every $24 \mathrm{~h}$, cultures were diluted into three new test-tubes containing casein medium with a dilution factor of $5,10,20$. The testtube with the highest dilution that showed significant growth was used in the next day to propagate the culture. Evolutionary improvement or loss of proteolytic activity were assayed every 4 days by comparing colony halo size on skim-milk plates for $\sim 100$ isolates (see next paragraph).

\section{Qualitative halo estimates on skim-milk plates}

Cultures were diluted onto LB plates and incubated for $16 \mathrm{~h}$ at $37^{\circ} \mathrm{C}$. The following morning, 100 colonies were picked and spotted on skim-milk plates (with wild-type (AEA101), and $\Delta l a s R$ (AEA102) as controls). The plates were incubated for $16 \mathrm{~h}$ at $30^{\circ} \mathrm{C}$ and the following morning colonies were screened for quorum-sensing activity (growth and proteolytic activity) by comparing the size of the formed halo with that of the controls.

\section{Elastase assay}

Elastase activity was quantified by a modification of a procedure described previously [36]. In brief, bacterial cultures were incubated with shaking at $37^{\circ} \mathrm{C}$ for $12 \mathrm{~h}$ in $0.5 \%$ CAA medium. Ten mg of Elastin-Congo Red (Sigma) and $1.01 \mathrm{~mL}$ of reaction buffer $(0.05 \mathrm{M}$ Tris-HCl buffer, $\mathrm{pH} 7.0,0.5 \mathrm{mM}$ $\mathrm{CaCl}_{2}$ ) were added to $15 \mathrm{~mL}$ glass tubes. After centrifugation of the culture, $10 \mu \mathrm{l}$ of supernatant was added to the glass tubes and the mixture was incubated at $37^{\circ} \mathrm{C}$ for $4 \mathrm{~h}$. The reaction was terminated by adding $100 \mu \mathrm{L}$ of $0.12 \mathrm{M}$ EDTA (pH 8.0). After centrifugation of the slurry, the absorbance at $495 \mathrm{~nm}$ of the supernatant was measured with a spectrophotometer zeroed on a control Elastin-Congo Red sample incubated without enzyme. As absorbance of $\operatorname{mexT}^{+}$strains was lower than that of the blank, the absorbance value of all strains was corrected by subtracting the mean absorbance level of the strain with lowest absorbance level.

\section{Relative growth and competition measurements in casein}

Relevant strains were transformed using pUB-GFP plasmid (Supplementary Table S2). Transformed strains were streaked from frozen stocks onto selective agar plates. After $16 \mathrm{~h}$ incubation at $37^{\circ} \mathrm{C}$, a single colony was picked and inoculated into $2 \mathrm{~mL}$ of a $0.5 \%$ CAA medium supplemented with carbenicillin. The colonies were cultured for $16 \mathrm{~h}$ at $37^{\circ} \mathrm{C}$ with agitation. The following morning, $\mathrm{OD}_{600}$ of cells was measured (usually 1-2) and cultures were diluted to an $\mathrm{OD}_{600}$ of 0.1 into $2 \mathrm{~mL}$ of $1 \%$ casein medium.
Prior to incubation, a sample from each culture was diluted into phosphate-buffered saline and cell count per unit time was determined using flow cytometer measurements of events expressing GFP. Cultures were then incubated at $37^{\circ}$ $\mathrm{C}$ with agitation for the indicated times. At each time point, cell counts were again measured by flow cytometry. Relative growth was calculated as the ratio of cell counts after and before incubation.

For each competition assay, relevant pairs of strains transformed with either the pUB-GFP or the pUB-BFP plasmids (Table S1), were streaked from frozen stocks onto selective agar plates and grown separately as described above. Strains were then diluted to an $\mathrm{OD}_{600}$ of 0.1 (unless otherwise stated) and mixed to the required relative frequencies. At time zero and at later indicated time points, flow cytometry was used to identify and quantify the number of BFP and GFP expressing cells. Each competition experiment was performed with switched markers and results shown are averages over multiple experiments, half with one marker combination and half with the other. It was clear from the experiments that the BFP plasmid had a slightly higher cost than the GFP plasmid (Fig. S4). Relative fitness of a focal strain over its co-cultured strain was calculated by dividing the relative frequency of the strains after incubation to that prior to incubation.

\section{Gene expression measurements in CAA and casein media}

For CAA gene expression experiments, overnight cultures of the relevant strains were diluted to an $\mathrm{OD}_{600}$ of 0.0005 into 96-well plate containing 0.5\% CAA medium supplemented with $300 \mu \mathrm{g} / \mathrm{mL}$ carbenicillin. Each well contained $100 \mu \mathrm{L}$ CAA medium and $120 \mu \mathrm{L}$ of mineral oil. The plate was incubated in a plate reader (2030 multilabel reader from PerkinElmer Victor X3) up to $72 \mathrm{~h}$ and wells were measured for $\mathrm{OD}_{600}$ and GFP levels every 15-20 minutes. Different repeats of the same experiment (both within and between days) were aligned by setting the time to $+3 \mathrm{~h}$ when their optical density read was at 0.1 . To allow for calculation of means and standard deviations, readouts were interpolated into constant timesteps, the dense measurement sampling ensures that interpolation does not affect the results. For casein medium, strains carrying the $\mathrm{P}_{\text {las }}$-GFP reporter were grown overnight in CAA medium and then diluted to an $\mathrm{OD}_{600}$ of 0.1 into casein medium. GFP expression measurements were taken at the indicated time points using flow cytometry.

\section{Flow cytometry}

All samples were run in a Gallios flow cytometer (Beckman-Coulter) equipped with four lasers $(405 \mathrm{~nm}, 488 \mathrm{~nm}$ co-linear with $561 \mathrm{~nm}, 638 \mathrm{~nm}$ ). The emission filters used 
Table 1 Whole-genome sequencing results of various strains in comparison with their parental strain

\begin{tabular}{|c|c|c|c|c|c|c|c|c|}
\hline \multirow[b]{2}{*}{ Strain } & \multirow[b]{2}{*}{ Ancestor } & \multirow[b]{2}{*}{ Tube (Day) ${ }^{\mathrm{a}}$} & \multicolumn{2}{|l|}{$p s d R$} & \multicolumn{2}{|l|}{$\operatorname{mexT}$} & \multirow[b]{2}{*}{ Additional genes affected } & \multirow[b]{2}{*}{$\mathrm{Cm}^{\mathrm{R}}$} \\
\hline & & & Allele & Effect & Allele & Effect & & \\
\hline AEA936 & $\Delta l a s R$ & $4(20)$ & T149A & $\mathrm{L} \rightarrow \mathrm{Q}$ & & & PA1097 (fleQ) & $-{ }^{c}$ \\
\hline AEA934 & $\Delta l a s R$ & $4(20)$ & T149A & $\mathrm{L} \rightarrow \mathrm{Q}$ & G827A & $G \rightarrow D$ & $\mathrm{PA} 3457^{\mathrm{b}}$ & - \\
\hline AEA937 & $\Delta l a s R$ & $4(20)$ & T149A & $\mathrm{L} \rightarrow \mathrm{Q}$ & G827A & $\mathrm{G} \rightarrow \mathrm{D}$ & PA0580 (ygjD) & - \\
\hline AEA938 & $\Delta l a s R$ & $4(20)$ & T149A & $\mathrm{L} \rightarrow \mathrm{Q}$ & G827A & $\mathrm{G} \rightarrow \mathrm{D}$ & PA3457 $7^{\mathrm{b}}, \mathrm{PA} 2094^{\mathrm{b}}$ & - \\
\hline AEA933 & $\Delta l a s R$ & $2(37)$ & G3A & M1I (Loss of start codon) & T722A & $\mathrm{L} \rightarrow \mathrm{Q}$ & & - \\
\hline AEA941 & $\Delta l a s R$ & $3(37)$ & A469C & $\mathrm{T} \rightarrow \mathrm{P}$ & $\mathrm{T}$ insertion at 547 & Frame-shift & psdR-dppA3 intergenic & - \\
\hline AEA942 & $\Delta l a s R$ & $3(37)$ & A469C & $\mathrm{T} \rightarrow \mathrm{P}$ & $\mathrm{T} 368 \mathrm{C}$ & $\mathrm{L} \rightarrow \mathrm{P}$ & $p s d R-d p p A 3$ intergenic & - \\
\hline AEA935 & WT & & G41A & $\mathrm{R} \rightarrow \mathrm{H}$ & C983A & $\mathrm{P} \rightarrow \mathrm{Q}$ & psdR-dppA3 intergenic & - \\
\hline AEA $325^{\mathrm{d}}$ & & & & & C712A & $\mathrm{P} \rightarrow \mathrm{T}$ & & - \\
\hline
\end{tabular}

Shown are the specific mutations in the genes $p s d R$ and mexT and their consequences in the protein sequence. Additional genes which acquired mutations are also shown. The last column indicates whether the strains can grow in $100 \mu \mathrm{g} / \mathrm{mL}$ of chloramphenicol

${ }^{a}$ The day the sample was taken is in parenthesis

${ }^{\mathrm{b}}$ Low coverage area/mixed reads-possible artifact

${ }^{\mathrm{c}}+$ and - Indicate whether the cell grew or did not grow on $100 \mu \mathrm{g} / \mathrm{mL}$ of chloramphenicol. A level in which the ancestors $\Delta l a s R$ and wild-type strain did grow

${ }^{\mathrm{d}}$ Kindly provided by the Banin lab

were: BFP - 450/50, GFP - 525/40. All samples were run at the "low" acquisition rate to reduce intra-sample variability. Events were discriminated upon the forward-scatter parameter. For each run, at least 100,000 cells were counted, and the total events analyzed was such that minority population was never below 1000 events.

\section{Next-generation genomic sequencing and analysis}

A total of nine strains were sequenced using deep sequencing. For each strain, an isolated colony was picked from an LB plate and inoculated into LB broth. The cultures were incubated for $16 \mathrm{~h}$ as outlined previously, and DNA was harvested with the Promega genomic DNA purification kit. Sequencing was done using the Technion sequencing services. All strains were pooled and ran on a single lane of a HighSeq illumina sequencer with a read length of $50 \mathrm{bp}$. Average coverage of each strain was $\sim 90 \times$. Analysis was done with the help of Professor Rotem Sorek, from the Weizmann Institute of Science, using a program that was developed at his Laboratory.

\section{Results}

\section{Directed evolution of $\Delta / a s R$ and wild-type PA01 strains in casein medium lead to reproducible consecutive mutations in the genes psdR and mexT}

It has been previously shown that growth in minimal medium with casein as a sole carbon source requires the quorum sensing-dependent secretion of proteases [18, 36]. We wondered whether a quorum-sensing mutant would be able to evolve cooperative growth in casein medium, despite possible exploitation by the non-evolved cells. To this aim, we inoculated four parallel lines with $\sim 10^{7}$ cells of a $\Delta$ lasR PAO1 strain (strain AEA102, Table S1). As no growth was initially observed, test-tubes were incubated until any signs of growth would appear. As a control, we also evolved a wild-type PAO1 strain (strain AEA101, Table S1), following cycles of growth and dilutions similarly to what was previously published $[18,19]$ (methods).

Strikingly, we found that both the $\Delta l a s R$ and wild-type genetic backgrounds evolved increased level of proteolytic activity. First, all four $\Delta l a s R$-containing test-tubes became opaque after 32-37 days of incubation, indicating increased casein hydrolysis activity and growth. The directed evolution experiments were terminated at that stage (methods) and isolates were picked from each replicate. The proteolytic activity of isolates was then assessed by their ability to form a degradation halo on skim-milk plates. We found that $>60 \%$ of the cells in each evolved population showed a substantial increase in their halo size compared with the halo of their $\Delta$ lasR parental strain. Second, we found that after several cycles of growth and dilution, the growth of the wild-type cells improved. Examination of isolates from the $7^{\text {th }}$ cycle revealed a high number ( $>50 \%$ ) of isolates with increased halo size compared with the halo of their parental wild-type strain.

To identify the genetic basis for the regain of proteolytic activity and ability to invade the population, we chose seven isolates with high proteolytic activity from three of the 
Table 2 Genotyping of mexT and $p s d R$ of 13 blindly chosen isolates from a single evolved line of the $\Delta$ lasR strain

\begin{tabular}{lllllll}
\hline Halo size phenotype & Number of isolates & $p s d R$ allele & Effect & mexT allele & Effect & $\mathrm{Cm}^{\mathrm{R}}$ \\
\hline Ancestral & 4 & Wild-type & & Wild-type & & + \\
Ancestral & 1 & C209T & $\mathrm{Q} \rightarrow$ Stop & Wild-type & & + \\
Ancestral & 1 & A529C & $\mathrm{T} \rightarrow \mathrm{P}$ & Wild-type & & + \\
Ancestral & 1 & A389C & $\mathrm{V} \rightarrow \mathrm{A}$ & Wild-type & & + \\
Evolved & 4 & R431C & $\mathrm{Y} \rightarrow \mathrm{S}$ & G502A & $\mathrm{E} \rightarrow \mathrm{K}$ & - \\
Evolved & 1 & $\mathrm{R} 431 \mathrm{C}$ & $\mathrm{Y} \rightarrow \mathrm{S}$ & A176C & $\mathrm{D} \rightarrow \mathrm{A}$ & - \\
Evolved & 1 & $\mathrm{R} 431 \mathrm{C}$ & $\mathrm{Y} \rightarrow \mathrm{S}$ & $\Delta(51-165)$ & Truncation & - \\
\hline
\end{tabular}

The first column indicates the halo size on skim-milk; Ancestral (small) or evolved (large). The last column indicates whether the strains can grow in $100 \mu \mathrm{g} / \mathrm{mL}$ of chloramphenicol
$\Delta$ las $R$ evolved lines and one isolate from the evolved wildtype line for deep sequencing, alongside their parental strains. We also sent for sequencing an additional wild-type PAO1 strain (AEA325, Table S1), which displayed an increased level of proteolytic activity compared with the one which we used to initiate directed evolution (Table 1). Strikingly, we found that all evolved isolates but one, contained mutations in two genes compared to their respective parental strains; $p s d R$ and mexT. Multiple different mutations in these two genes were observed, including in strains obtained from the same evolved testtube. Two isolates from the same test-tube contained the same psdR mutation, but different mutations in mexT, suggesting that the $p s d R$ mutation arose first. Notably, the highly proteolytic PAO1 strain (strain AEA325, Table S1) carried only a single mutation in the mex $T$ gene compared with the wild-type strain we used in our directed evolution experiment, which displayed weaker proteolytic activity (AEA101, Table S1).

In addition to mutations in $p s d R$ and mexT, further mutations were found in other loci (Table 1). Two mutations were found in the intergenic region downstream of $p s d R$ and upstream of the $d p p A$ gene. These mutations are most likely functionally related to $p s d R$. Other mutated genes appeared only in single isolates. One isolate did not carry a mutation in mex $T$, but rather in the gene fle $Q$ [41]. We did not further characterize this mutation.

With a focus on $p s d R$ and mexT, we Sanger-sequenced those loci for 13 randomly chosen isolates from a single evolved $\Delta$ lasR line (Table 2). We found that 4 out of 13 isolates had wild-type alleles in both loci. All other nine strains contained one of four different mutations in $p s d R$, including one nonsense mutation. Mutations in $\operatorname{mex} T$ were only found in a psdR mutant background. Different mexT mutations, including a large deletion, were found with the same $p s d R$ mutation. Altogether, the deep sequencing and Sanger sequencing results support a model in which $p s d R$ mutations have evolved first, followed by mutations in mexT. The selection of multiple different mutations in each of the genes, as well as the nature of some of the mutations (e.g., frameshifts, nonsense and start codon elimination, Tables 1,2), suggests that they resulted in loss of function of the corresponding genes.

PsdR is a negative regulator of genes involved in the intake and degradation of short peptides [42]. Recently, it was shown that growth in casein medium leads to strong selection for $p s d R$ null mutations in both a cooperator and an evolved lasR mutant [43]. It was also shown that the $p s d R$ mutation does not contribute directly to the secretion of proteases and is selected for its associated private benefit. We therefore focused on understanding the role of mexT in the evolution of regained cooperation.

MexT is a transcriptional regulator of a variety of genes [44]. This regulatory function includes the activation of the mexEF-oprN operon, which codes for an RND family multidrug-resistance pump critical for resistance to various substances, including the antibiotic chloramphenicol $(\mathrm{Cm})$ [45, 46]. Loss of MexT activity should therefore directly lead to reduction in mexEF-oprN expression, which will result in $\mathrm{Cm}$ sensitivity. Previous works have identified significant diversity of mexT and mexEF-oprN alleles in PAO1 "wild-type" strains [47]. We therefore refer to all strains below as either mex $T^{+}$or mexT and avoid using the term "wild-type".

The MexEF-OprN pump was shown to reduce the Rhl and PQS quorum-sensing activity [44, 48, 49], most likely through the export of HHQ (4-hydroxy-2-heptylquinoline), which serves both as a direct signal and an intermediate in the production of the PQS signal molecule [50]. To determine the effect of the mexT mutations on mexEF-opr $N$ expression, we transformed the ancestral mex $T^{+}$and $\Delta$ lasR;mex $T^{+}$strains, as well as several evolved isolates, with a GFP transcriptional reporter plasmid for the mexEF-oprN operon expression (methods). In agreement with a loss-of-function effect of mexT, the GFP expression level of the mexT mutant strains was significantly lower than that of the strains coding for the mexT wild-type allele when grown in casamino-acids (Fig. 1a, $p<10^{-3}$ for all comparisons of the same genetic background, two sample $t$ test for average of all samples from the last $2 \mathrm{~h}$ of sampling, $N=4$ for each comparison). 


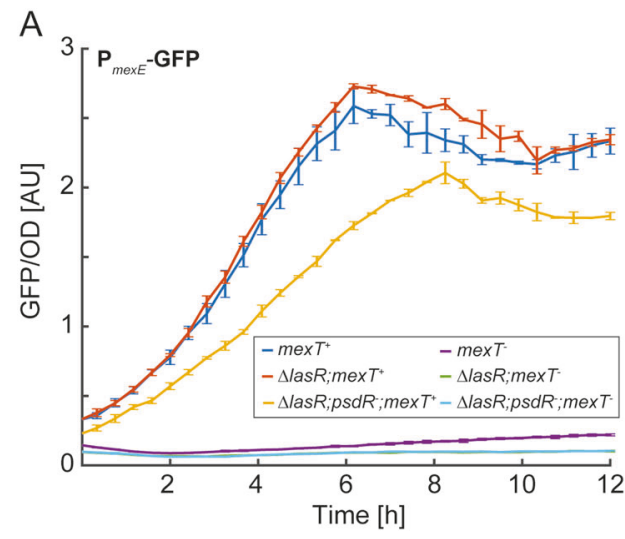

Fig. 1 Characteristics of evolved mutants. a Activity of a GFP transcriptional reporter for the mexE promoter as a function of time. Activity is measured as fluorescence level divided by optical density. Results are shown for six genotypes grown in casamino acid medium; $\operatorname{mexT}^{+}$(blue), $\Delta$ lasR;mex $T^{+}$(red), $\Delta$ lasR;psdR ${ }^{-} ; m e x T^{+}$(orange), mexT (purple); $\Delta$ lasR; mexT (green) and $\Delta l a s R ; p s d R^{-} ; \operatorname{mexT}$ (cyan). b, $\mathbf{c}$ Growth in casein medium of seven relevant genotypes. Growth was measured as the ratio between cell counts after and before

Consistently, we also found that all isolates that coded for a mutated mexT became sensitive to $\mathrm{Cm}$ at a concentration of $100 \mu \mathrm{g} / \mathrm{mL}$, to which the strains coding for the mexT wild-type allele were resistant (Tables 1,2). Notably, the only evolved $\Delta$ lasR strain, which did not acquire a mexT mutation, but rather an $f l e Q$ mutation, also showed low $\mathrm{Cm}$ resistance (Table 1), suggesting that regain of cooperation in this strain is also mediated by MexEF-OprN inactivation. This is an unknown regulatory link, but we did not further characterize it in this work.

\section{Growth in casein reflects the functions of the mexT and $p s d R$ mutations}

To better estimate the contribution of each mutation to cooperative growth in casein medium, we assayed the ability of each strain to clonally grow in casein. Generally, we found that $\Delta l a s R$-derived strains grew significantly slower than $\operatorname{las}^{+}$-derived strains when inoculated at the same initial density (Fig. 1b, Fig. S1). As cooperative growth depends on initial density [7,25], we compensated for this growth difference by growing las $^{+}$-derived strains from an initial inoculation, which is a factor of 10 lower than that of the $\Delta$ lasR-derived strains (Fig. 1c). In agreement with their qualitative effect on halo size (Fig. S2), we found that the $p s d R^{-}$allele did not significantly increase growth of the $\Delta l a s R$ mutant over an incubation period of 24 or $48 \mathrm{~h}$ ( $p>0.6$, two sampled $t$ test). In contrast, the mexT allele showed a significant increase in growth $(p<0.02$ after $48 \mathrm{~h}$, two sampled $t$ test, $N=12$ ). The two mutations synergistically interacted to further increase growth in a $p s d R^{-}$;mexT double mutant strain (Fig. $1 \mathrm{~b}, p<0.03$ two

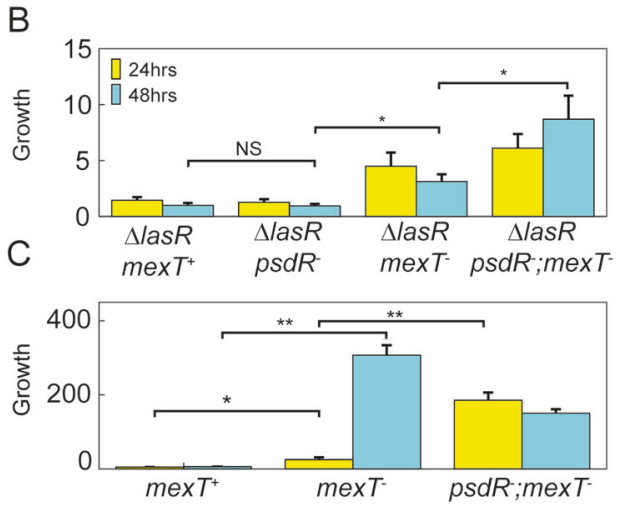

the indicated incubation intervals using flow cytometry. Shown are results for $\mathbf{b} \Delta$ las $R$ background strains inoculated at an initial optical density of 0.1 , c las $R^{+}$background strains, inoculated at an initial optical density of 0.01 . Error bars represent standard error of three or more independent repeats on different days. Exact genotypes are provided in Supp. Tables S1, S2. NS—non significant. * $-p$ value $<$ $0.05, * * \ldots p$ value $<10^{-3}$. (color figure online)

sampled $t$ test, $N=12$ ). Similar results, but with higher growth rate, were obtained with the las $^{+}$background (Fig. 1c).

\section{The mext mutation has a strong effect on protease activity and other Rhl-dependent genes in $\Delta$ lasR and Ias $^{+}$backgrounds}

We wanted to verify that the mex $T^{-}$alleles lead to increased protease activity and a corresponding increase in protease gene expression in the $\operatorname{las}^{+}$and $\Delta$ las $R$ mutant backgrounds. To this aim, we measured the protease activity of the various strains when grown in minimal medium using the elastin-congo red proteolysis assay (Fig. 2a). In addition, we compared halo sizes of all strains on skim-milk plates (Fig. S2). In both assays the mexT allele dramatically increased proteolytic activity, whereas the $p s d R^{-}$ allele had a negligible effect.

Next, we monitored protease expression in casein by measuring gene expression of the major protease gene, las $B$. We transformed the $\Delta l a s R, \Delta l a s R ; p s d R^{-}$, and $\Delta l a s R ; p s d R^{-}$; mex $T^{-}$strains with a transcriptional GFP reporter for las $B$ and measured its activity during growth in casein using flow cytometry (Fig. 2b, methods). We found that the $\Delta l a s R$, and $\Delta$ lasR;psd $R^{-}$strains showed no las $B$ reporter expression throughout the experiment. In contrast, GFP expression was observed for the $\Delta l a s R ; p s d R^{-}$;mexT $T^{-}$strain $\left(p<2 \times 10^{-3}\right.$ for increased response compared with $\Delta l a s R$ at $48 \mathrm{~h}$, two sampled $t$ test, $N=6$ ). This expression was high upon initial inoculation into casein medium, reduced and then increased again during growth, in a manner corresponding to quorum sensing-dependent regulation. This observation further 
A

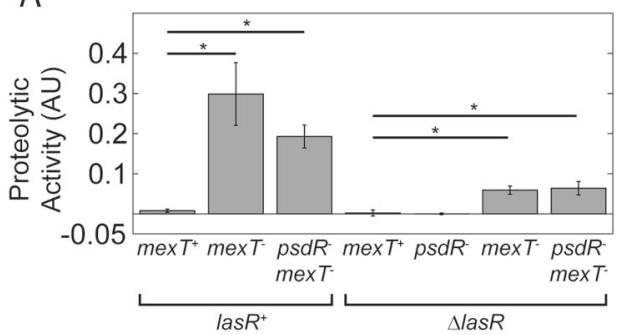

B

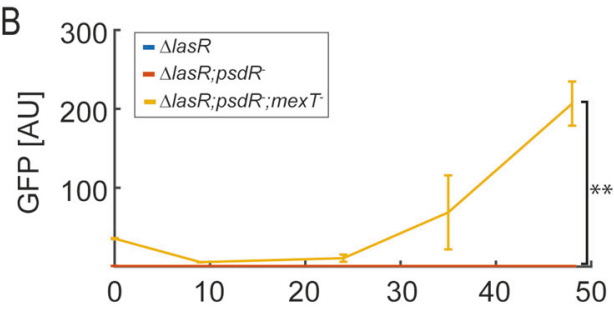

C
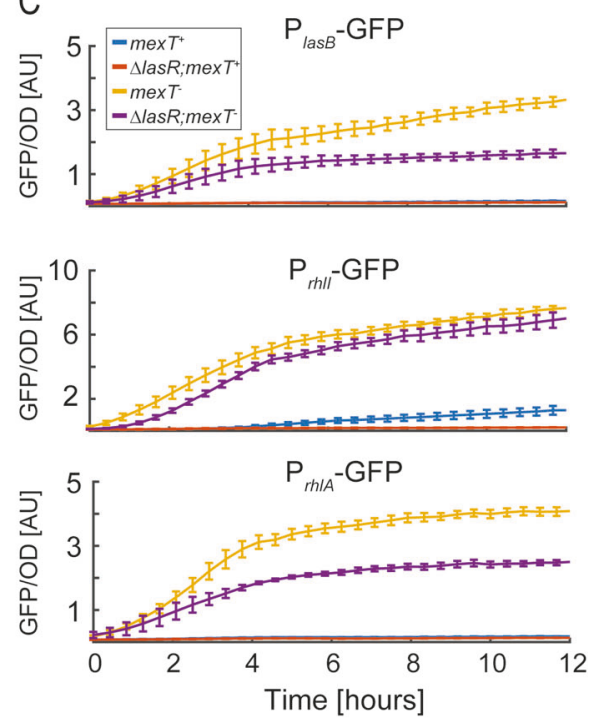

of time of GFP transcriptional reporters for lasB (top panel), rhlA (middle panel), and rhlI (bottom panel). Results in each panel are shown for four genotypes; mexT $T^{+}$(light blue), $\Delta$ lasR (red), mexT (orange), and $\Delta$ lasR; mexT (magenta). Error bars represent standard error of three or more independent repeats on different days. Exact genotypes are provided in Supplementary Tables S1, S2. *-p value $<$ $0.05, * *-p$ value $<2 \times 10^{-3}$. (color figure online)

activity of a pqsA GFP transcriptional reporter in all four strain combinations of the lasR and mexT alleles (Fig. S3). We found that the mexT $T^{+}$allele showed weak pqs expression in both $l a s R^{+}$and $\Delta l a s R$ backgrounds. The $m e x T^{-}$allele dramatically increased expression in both backgrounds $\left(p<10^{-5}\right.$ in both cases, $\left.N=10\right)$ but was twofold stronger in the las $R^{+}$background $\left(p<10^{-5}, N=\right.$ 10). This indicates that las $R$ deletion, while affecting PQS response, does not eliminate the dependence on MexT activity.

\section{The mexT allele enhances cooperation through inactivation of MexEF-OprN, but increases private benefit in a MexEF-OprN-independent manner}

A notable problem in the evolutionary regain of cooperative behaviors is their susceptibility to exploitation by ancestral non-cooperative strains $[24,26]$. Specifically, for a mexT ${ }^{-}$ strain to invade into its ancestor $m e x T^{+}$strain, its direct fitness should be increased irrespective of its ability to increase public goods secretion.

Previous work on MexT has ascribed the increase in Rhl activity primarily to the inactivation of the MexEF-OprN pump [54]. This suggests that the increase in public goods production observed in our evolutionary experiments could have also arisen through mutations to the mexEF-oprN. Nevertheless, all cooperation-enhancing mutations we 

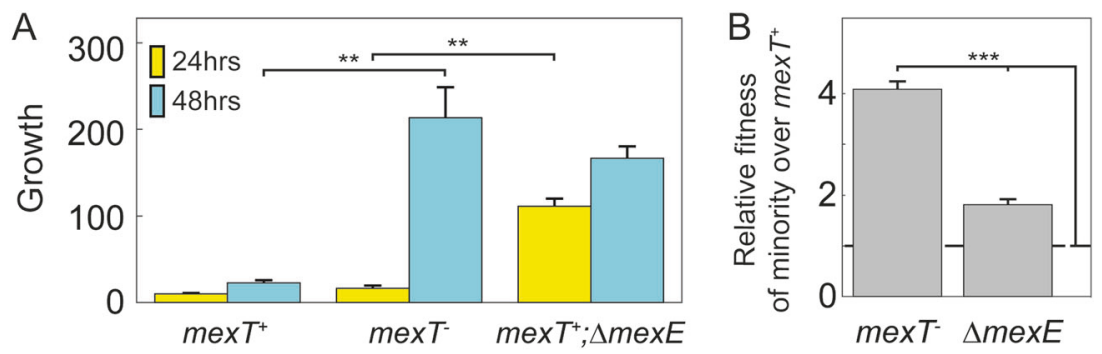

Fig. 3 Growth $\mathbf{a}$ and selection in co-culture $\mathbf{b}$ of different mex variants. a Growth after 24 and $48 \mathrm{~h}$ of the efflux inactive strains mexT ; mex $E^{+}$, $m e x T^{+} ; \Delta m e x E$, and the efflux active $m e x T^{+} ; m e x E^{+}$strain. Growth is defined as in Fig. 1a, b. Averages and standard errors are calculated for multiple repeats performed on three separate days. b The strains $m e x T^{-} ; m e x E^{+}$(AEA1174) and $m e x T^{+} ; \Delta \operatorname{mexE}$ (AEA1176) were separately co-cultured as minorities with the efflux active strain $m e x T^{+} ;$mexE $^{+}$(AEA1175). Shown is the relative fitness of each minority strain over the majority strain. Relative fitness is calculated by dividing the relative frequency of two co-cultured strains at the end

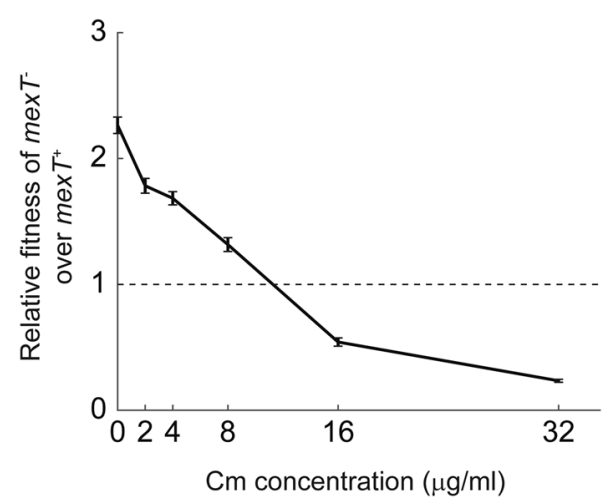

Fig. 4 Relative fitness of a minority $\operatorname{mex} T^{-}(\sim 5 \%$, strain AEA1174) in a majority of $\operatorname{mexT}^{+}$(strain AEA1175) after $48 \mathrm{~h}$ of growth in casein medium as a function of different concentrations of Chloramphenicol. All samples (including the no antibiotic sample) have the same level of ethanol carrier. Relative fitness is defined as in Fig. 3 caption. Error bars represent the standard error of the mean of six biological repeats, three with the minority marked with BFP and three with the minority marked with GFP. See Fig. S4 for separate graphs of the two markers.

identified were in the mexT gene, despite its small target size $(\sim 1 \mathrm{~kb})$ compared with the mexEF-oprN operon $(\sim 6 \mathrm{~kb})$. This led us to suspect that null mutation of mexT may mediate a private benefit to the cells in a mexEF-oprNindependent manner.

In order to assess this hypothesis, we obtained an iso-

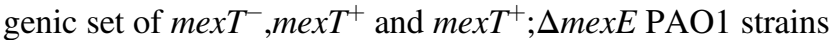
(strains AEA1174, AEA1175, AEA1176, Table S1) [48].

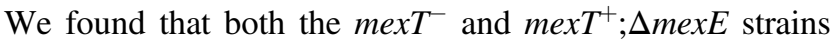
grew much better than the isogenic mexT $T^{+}$strain in casein medium (Fig. 3a, $p<10^{-3}$ for $\operatorname{mexT} T^{-}$over $\operatorname{mex} T^{+}$after $48 \mathrm{~h}$

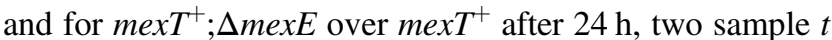
test, $N=10$ ). Interestingly, the $\operatorname{mex} T^{+} ; \Delta \operatorname{mexE}$ strain grew of growth (after $48 \mathrm{~h}$ ) by their relative initial frequency in the coculture. A value of 1 indicates no fitness advantage. Initial frequency of the efflux inactive strains was set to $\sim 5 \%$. Co-cultured strains coded for a GFP and BFP constitutive reporters. Results are the summary of 18 co-culture experiments for each co-cultured pair, in half of the repeats the BFP was expressed in the majority strain and in half it was expressed in the minority strain. a, b Error bars represent the standard error of the mean. $* * p$ value $<10^{-3}$, *** $p$ value $<10^{-6}$. b Significance is also with respect to deviation from a value of one (no fitness difference). (color figure online)

significantly faster than the mexT $T^{-}$strain $\left(p<10^{-5}\right.$ after 24 h, two sample $t$ test, $N=10$ ).

Next, we performed competition experiments between a majority of the proteolytically inactive $\operatorname{mexT}^{+}$strain and a minority of either one of the proteolytically active strains (mexT or mexT $T^{+} ; \Delta$ mexE) separately. (Fig. 3b, methods). We found that the mexT $T^{-}$strain was able to increase its frequency by a factor of $\sim 4$ after 2 days of growth $\left(p<10^{-10}, t\right.$ test, $N$ $=26$ ). This validated the evolutionary trajectory obtained in the evolution experiments and demonstrated the strong private benefit to the mutant strain. In contrast, the frequency of the $m e x T^{+} ; \Delta$ mexE strain increased only by a factor of 1.7 over two days of incubation $\left(p<10^{-7}, t\right.$ test, $\left.N=26\right)$, suggesting that this mutation harbors a smaller private benefit to the cells than the mexT $T^{-}$null allele $\left(p<10^{-10}, t\right.$ test). These results confirmed our hypothesis that a mex $T^{-}$null allele provides a strong private benefit to its carrier. The difference between the invasions of the two mex mutants suggests that much of this private benefit is independent of MexEF-OprN activity (see discussion).

\section{The adaptive advantage of mexT null alleles is eliminated in the presence of chloramphenicol}

Finally, the selection for a mex $T^{-}$null allele in an environment requiring quorum-sensing-dependent cooperation is opposite to the selection imposed by antibiotics for which resistance is mediated by the MexEF-OprN pump. This implies that selection for antibiotic resistance would prevent the regain of quorum-sensing activity and cooperation through inactivation of the MexEF-OprN pump. To further study this effect, we repeated the casein medium competition experiment between a minority of mexT strain and a majority of mexT $^{+}$strain in the presence of varying 
concentrations of $\mathrm{Cm}$ (Fig. 4). We found that the mexT relative fitness over the $m e x T^{+}$strain was reduced with increased levels of $\mathrm{Cm}$, becoming negative for $\mathrm{Cm}$ concentration larger than $\sim 10 \mu \mathrm{g} / \mathrm{mL}$. This concentration is substantially lower than the observed MIC of $\mathrm{Cm}$ for the $m e x T^{-}$strain, which we found to be $\sim 64 \mu \mathrm{g} / \mathrm{mL}$ (methods).

\section{Discussion}

Evolutionary regain of cooperation is hampered by the ability of non-mutant cells to exploit the evolved cooperator $[24,26]$. In the context of the evolutionary regain of quorum-sensing cooperation, exploitation is accompanied by the additional constrain imposed by the low levels of signaling molecules produced by the small minority of the evolved quorum-sensing cooperators $[29,30]$. These limitations were proposed to improve the effectiveness of antiquorum-sensing drugs [28]. Here, we found that a las $R$ deletion mutant, has evolved increased cooperation in casein medium through null mutations in mexT. Similar selection also occurred in a wild-type, $\operatorname{las}^{+}$, background. The mexT mutations arose after the selection of mutations in the $p s d R$ gene. $p s d R$ null mutants were previously shown to increase peptide utilization in $P$. aeruginosa [43].

The roles of MexT and the MexEF-OprN pump in the regulation of quorum-sensing activity have been analyzed in several previous works [44, 48, 49]. In addition, null mutations in mexT or mexEF-oprN are selected during clinical infections [47, 55] and lab culturing [47], leading to significant allelic diversity in these genes in clinical isolates and the PAO1 lab strain [45]. Despite the extensive literature on the subject, the interplay between the effects of mexT and mexEF-oprN on social and asocial sources of selection were not previously studied.

We found that the mexT null mutations increased the level of cooperative growth in both the $\Delta$ las $R$ and $l a s R^{+}$ backgrounds (Fig. 1). This increase is correlated with altered expression of Rhl-dependent genes in the $\operatorname{mexT} T^{-}$ mutants (Fig. 2). We note, however, that the changes in gene expression in casamino acid medium (Fig. 2b-d), do not perfectly match those observed for growth in casein (Fig. 1a, b). Although both gene expression and growth results displayed a strong dependence on allelic variation in mexT, the effects of variation in the las $R$ locus had a smaller effect in the gene expression assays than their effect on growth in casein. This may stem from the relative dependence of other secreted proteases on LasR and RhIR or from the different growth medium used in the two experiments.

To better understand the interplay between private and cooperative effects of the mexT $T^{-}$allele, we compared the cooperative growth and competitive fitness of the mexT and $\operatorname{mex} T^{+} ; \Delta \operatorname{mexE}$ strains (Fig. 3). Although both strains showed increased clonal growth and a competitive advantage over the $\operatorname{mexT}^{+}$strain in casein medium, they differed in the relative levels of these two traits. The mexT strain showed a higher competitive advantage over the $\operatorname{mex} T^{+}$ strain than that of the mexT $T^{+} ; \Delta \operatorname{mexE}$ strain (explaining its selection, Fig. 3b). In contrast, the $\operatorname{mexT}^{+} ; \Delta$ mexE strain had an increased level of clonal growth (Fig. 3a). It was previously shown that the mexT $T^{-}$strain does not show any significant expression of the mexEF-oprN pump [56]. Therefore, the effects on both cooperative growth and competitive advantage, most likely arise through a MexEFOprN-independent activity of MexT. A previous work identified several MexEF-OprN-independent functions of MexT, including the repression of the type III secretion systems, pyocyanin production, and surface attachment [54]. It is unclear whether the expression of these or other MexT-dependent traits could explain the observed differences.

We note that the mexE deletion was also beneficial to the cells, though to a lesser degree. This indicates that private benefit is also achieved via inactivation of the pump. This benefit may stem from several alternative sources. First, the MexEF-OprN pump activity was shown to affect proton gradient across the cell membrane, which may have adverse effects on growth [57]. Second, the private benefit may be mediated by PQS-related activities. Finally, the effect may be directly related to Rhl-associated private goods (e.g., cyanide resistance [58]) or to some level of privatization of exoprotease activity, which will lead to selection for exoprotease-secreting cells at low density, as was previously shown for yeast invertase secretion [25].

It is surprising that two mutations accumulated in the same cell during the evolution of the $\Delta$ las $R$ mutant despite the lack of apparent growth over the $>4$ weeks of incubation. An initial population death phase in which $p s d R$ null alleles were selected for their reduced death rate may partially explain this evolutionary trajectory. The reduced population size would have allowed for a larger number of cell divisions over which the emergent mexT mutant alleles could have subsequently increase their frequency. Initially, the selection of the mexT mutants may depend mostly on their private benefit. Public goods secretion later leads to further growth and selection. Private and public benefits may therefore synergistically contribute to selection dynamics.

Pleiotropic genetic effects that link private and cooperative benefits have been previously observed in P. aeruginosa quorum-sensing [19, 58] and other cooperating microorganisms [59, 60]. In general, these pleiotropic couplings are expected to allow the reintroduction of cooperation into a cheater dominated population $[23,24]$ and slow down the emergence of cheaters in cooperator populations [59]. On a longer time-scale, pleiotropy on its own would not preclude the emergence of cheaters, as 
further mutations may uncouple the cooperative and private traits [61]. Specifically, in our case, while the initial evolution of the wild-type $\left(\operatorname{mex} T^{+}\right)$led to increased private and cooperative benefits through the effects of the $p s d R$ and mexT null alleles, further selection led to the emergence of lasR cheater mutants [18, 43]. In other cases, such as pyoverdine based cooperation, lack of pleiotropic dependence leads to a complete block on the evolutionary regain of cooperation [26].

Previous works on the evolution of quorum-sensing cooperation in casein and other protein-based media did not identify mexT as a locus affecting cooperation. This is most likely because a $\operatorname{mex} T^{-}$allele was already fixed in the original genetic background of the starting cultures. We have verified this specifically with the authors of a previous work, which identified mutations in the $p s d R$ locus ([43], K. Asfahal, private communication). The presence of the mexT ${ }^{+}$allele in some PAO1 strains (including the one used by us for directed evolution) and more so in the $\Delta l a s R$ strain, where some of its phenotypes are masked, further demonstrates the pervasiveness of this allelic variation and the need to verify its status when considering quorum-sensing mutant phenotype [47].

Finally, RND family multidrug-resistant pumps have a crucial effect on the quorum-sensing network of $P$. aeruginosa. In addition to the MexEF-OprN pump discussed here, the MexAB-OprM pump exports the Las signal, 3-oxo-C12 homoserine lactone [62, 63], and its overexpression was shown to reduce quorum-sensing activity [64]. MexABOprM is also regulated by quorum-sensing, forming a feedback on quorum-sensing activity [49]. RND family of multidrug-resistance pumps appear to play a similar role in other Gram-negative bacteria with quorum-sensing systems, which utilize long-chained homoserine lactone autoinducers [65]. In all these cases, strong activation of the pumps may be detrimental to quorum-sensing activity, but necessary for antibiotic resistance. Antibiotic selection is therefore expected to greatly modify the shape and evolution of the quorum-sensing activation and cooperation landscape. As we showed in our work, even sub-MIC concentrations of $\mathrm{Cm}$ prevented the selection of the mexT allele and led to a reduction in the level of cooperativity (Fig. 4). It would be of interest to identify genetic mechanisms that help resolve this conflict when both antibiotic resistance and quorum-sensing directed cooperation are being selected for.

Acknowledgements This work was supported by European Research Council grant \#281301 (K.Z, I.S, S.O.B) and the Israeli Science Foundation grant \#418/15 (R.D.O, S.O.B). We are thankful to Peter Greenberg and Ajai Dandekar for their help with establishing basic $P$. aeruginosa assays. We thank Udi Banin and Thilo Köhler for providing plasmids, strains, and advice. We also thank Rotem Sorek for help with the analysis of genome sequences.

\section{Compliance with ethical standards}

Conflict of interest The authors declare that they have no conflict of interest.

Open Access This article is licensed under a Creative Commons Attribution 4.0 International License, which permits use, sharing, adaptation, distribution and reproduction in any medium or format, as long as you give appropriate credit to the original author(s) and the source, provide a link to the Creative Commons license, and indicate if changes were made. The images or other third party material in this article are included in the article's Creative Commons license, unless indicated otherwise in a credit line to the material. If material is not included in the article's Creative Commons license and your intended use is not permitted by statutory regulation or exceeds the permitted use, you will need to obtain permission directly from the copyright holder. To view a copy of this license, visit http://creativecommons. org/licenses/by/4.0/.

\section{References}

1. Griffin AS, West SA, Buckling A. Cooperation and competition in pathogenic bacteria. Nature . 2004;430:1024-7.

2. Xavier JB, Foster KR. Cooperation and conflict in microbial biofilms. Proc Natl Acad Sci. 2007;104:876-81.

3. West SA, Diggle SP, Buckling A, Gardner A, Griffin AS. The social lives of microbes. Annu Rev Ecol Evol Syst. 2007;38:53-77.

4. Waters CM, Bassler BL. Quorum sensing: cell-to-cell communication in bacteria. Annu Rev Cell Dev Biol. 2005;21:319-46.

5. Schuster M, Joseph Sexton D, Diggle SP, Peter Greenberg E. Acyl-homoserine lactone quorum sensing: from evolution to application. Annu Rev Microbiol. 2013;67:43-63.

6. Hense BA, Kuttler C, Muller J, Rothballer M, Hartmann A, Kreft J-U. Does efficiency sensing unify diffusion and quorum sensing? Nat Rev Micro. 2007;5:230-9.

7. Darch SE, West SA, Winzer K, Diggle SP. Density-dependent fitness benefits in quorum-sensing bacterial populations. Proc Natl Acad Sci. 2012;109:8259-63.

8. Pai A, Tanouchi Y, You L. Optimality and robustness in quorum sensing (QS)-mediated regulation of a costly public good enzyme. Proc Natl Acad Sci. 2012;109:19810-5.

9. Lee J, Zhang L. The hierarchy quorum sensing network in Pseudomonas aeruginosa. Protein Cell. 2015;6:26-41.

10. Diggle SP, Winzer K, Chhabra SR, Worrall KE, Cámara M, Williams $\mathrm{P}$. The Pseudomonas aeruginosa quinolone signal molecule overcomes the cell density-dependency of the quorum sensing hierarchy, regulates rhl-dependent genes at the onset of stationary phase and can be produced in the absence of LasR. Mol Microbiol. 2003;50:29-43.

11. Pesci EC, Pearson JP, Seed PC, Iglewski BH. Regulation of las and rhl quorum sensing in Pseudomonas aeruginosa. J Bacteriol. 1997; 179:3127-32.

12. Schuster M, Peter Greenberg E. A network of networks: quorumsensing gene regulation in Pseudomonas aeruginosa. Int $\mathrm{J}$ Med Microbiol. 2006;296:73-81.

13. McKnight SL, Iglewski BH, Pesci EC. The Pseudomonas quinolone signal regulates rhl quorum sensing in Pseudomonas aeruginosa. J Bacteriol. 2000;182:2702-8.

14. Pearson J, Pesci E, Iglewski B. Roles of Pseudomonas aeruginosa las and rhl quorum-sensing systems in control of elastase and rhamnolipid biosynthesis genes. J Bacteriol. 1997;179: 5756-67. 
15. Schuster M, Lostroh $\mathrm{CP}$, Ogi $\mathrm{T}$, Greenberg E. Identification, timing, and signal specificity of Pseudomonas aeruginosa quorum-controlled genes: a transcriptome analysis. J Bacteriol. 2003;185:2066-79.

16. Diggle SP, Griffin AS, Campbell GS, West SA. Cooperation and conflict in quorum-sensing bacterial populations. Nature. 2007;450:411-4.

17. Pollitt EJ, West SA, Crusz SA, Burton-Chellew MN, Diggle SP. Cooperation, quorum sensing, and evolution of virulence in Staphylococcus aureus. Infect Immun. 2014;82:1045-51.

18. Sandoz KM, Mitzimberg SM, Schuster M. Social cheating in Pseudomonas aeruginosa quorum sensing. Proc Natl Acad Sci. 2007;104:15876-81.

19. Dandekar AA, Chugani S, Greenberg EP. Bacterial quorum sensing and metabolic incentives to cooperate. Science. 2012;338:264-6.

20. Feltner JB, Wolter DJ, Pope CE, Groleau M-C, Smalley NE, Greenberg EP, et al. LasR variant cystic fibrosis isolates reveal an adaptable quorum-sensing hierarchy in Pseudomonas aeruginosa. mBio. 2016;7. e01513-16.

21. Rumbaugh K, Diggle S, Watters C, Ross-Gillespie A, Griffin A, West S. Quorum sensing and the social evolution of bacterial virulence. Curr Biol. 2009;19:341-5.

22. Köhler T, Buckling A, van Delden C. Cooperation and virulence of clinical Pseudomonas aeruginosa populations. Proc Natl Acad Sci. 2009;106:6339-44.

23. Foster K. The Phoenix effect. Nature . 2006;441:18.

24. Fiegna F, Yu Y-TN, Kadam SV, Velicer GJ. Evolution of an obligate social cheater to a superior cooperator. Nature. 2006;441:310-4.

25. Gore J, Youk H, van Oudenaarden A. Snowdrift game dynamics and facultative cheating in yeast. Nature. 2009;459:253-6.

26. Granato ET, Kümmerli R. The path to re-evolve cooperation is constrained in Pseudomonas aeruginosa. BMC Evol Biol. 2017;17:214.

27. Defoirdt T, Boon N, Bossier P. Can bacteria evolve resistance to quorum sensing disruption? PLoS Pathog. 2010;6:e1000989.

28. Allen RC, Popat R, Diggle SP, Brown SP. Targeting virulence: can we make evolution-proof drugs? Nat Rev Microbiol. 2014;12:300-8.

29. Mellbye B, Schuster M. The sociomicrobiology of antivirulence drug resistance: a proof of concept. mBio. 2011;2:e00131-11.

30. Gerdt JP, Blackwell HE. Competition studies confirm two major barriers that can preclude the spread of resistance to quorum-sensing inhibitors in bacteria. ACS Chem Biol. 2014;9:2291-99.

31. Köhler T, Perron GG, Buckling A, Van Delden C. Quorum sensing inhibition selects for virulence and cooperation in Pseudomonas aeruginosa. PLoS Pathog. 2010;6:e1000883.

32. Maeda T, García-Contreras R, Pu M, Sheng L, Garcia LR, Tomás $\mathrm{M}$, et al. Quorum quenching quandary: resistance to antivirulence compounds. ISME J. 2012;6:493-501.

33. Steindler L, Bertani I, De Sordi L, Schwager S, Eberl L, Venturi V. LasI/R and RhlI/R quorum sensing in a strain of Pseudomonas aeruginosa beneficial to plants. Appl Environ Microbiol. 2009;75:5131-40.

34. Cabeen MT. Stationary phase-specific virulence factor overproduction by a lasR mutant of Pseudomonas aeruginosa. PLoS ONE. 2014;9:e88743.

35. Medina G, Juárez K, Díaz R, Soberón-Chávez G. Transcriptional regulation of Pseudomonas aeruginosa rhlR, encoding a quorum-sensing regulatory protein. Microbiology. 2003; 149:3073-81.

36. Van Delden C, Pesci EC, Pearson JP, Iglewski BH. Starvation selection restores elastase and rhamnolipid production in a
Pseudomonas aeruginosa quorum-sensing mutant. Infect Immun. 1998;66:4499-502.

37. Schweizer HP. Alielic exchange in Pseudomonas aeruginosa using novel ColE1-type vectors and a family of cassettes containing a portable oriT and the counter-selectable Bacillus subtilis sacB marker. Mol Microbiol. 1992;6:1195-204.

38. Miller WG, Leveau JHJ, Lindow SE. Improved gfp and inaZ broad-host-range promoter-probe vectors. Mol Plant Microbe Interact. 2000;13:1243-50.

39. Davies DG,Parsek MR,Pearson JP,Iglewski BH,Costeron Jt, Greenberg E. The involvement of cell-to-cell signals in the development of a bacterial biofilm. Science. 1998;280:295-8.

40. Xavier JB, Kim W, Foster KR. A molecular mechanism that stabilizes cooperative secretions in Pseudomonas aeruginosa. Mol Microbiol. 2011;79:166-79.

41. Hickman JW, Harwood CS. Identification of FleQ from Pseudomonas aeruginosa as ac-di-GMP-responsive transcription factor. Mol Microbiol. 2008;69:376-89.

42. Kiely PD, O'Callaghan J, Abbas A, O'Gara F. Genetic analysis of genes involved in dipeptide metabolism and cytotoxicity in Pseudomonas aeruginosa PAO1. Microbiology . 2008;154:2209-18.

43. Asfahl KL, Walsh J, Gilbert K, Schuster M. Non-social adaptation defers a tragedy of the commons in Pseudomonas aeruginosa quorum sensing. ISME J. 2015;9:1734-46.

44. Tian Z-X, Fargier E, Mac Aogáin M, Adams C, Wang Y-P, O'gara F. Transcriptome profiling defines a novel regulon modulated by the LysR-type transcriptional regulator MexT in Pseudomonas aeruginosa. Nucleic Acids Res. 2009;37: 7546-59.

45. Maseda H, Saito K, Nakajima A, Nakae T. Variation of the mexT gene, a regulator of the MexEF-oprN efflux pump expression in wild-type strains of Pseudomonas aeruginosa. FEMS Microbiol Lett. 2000;192:107-12.

46. Maseda H, Yoneyama H, Nakae T. Assignment of the substrateselective subunits of the MexEF-OprN multidrug efflux pump of Pseudomonas aeruginosa. Antimicrob Agents Chemother. 2000;44:658-64.

47. Luong PM, Shogan BD, Zaborin A, Belogortseva N, Shrout JD, Zaborina $\mathrm{O}$, et al. Emergence of the P2 phenotype in Pseudomonas aeruginosa PAO1 strains involves various mutations in mexT or mexF. J Bacteriol. 2014;196:504-13.

48. Köhler T, van Delden C, Curty LK, Hamzehpour MM, Pechere JC. Overexpression of the MexEF-OprN multidrug efflux system affects cell-to-cell signaling in Pseudomonas aeruginosa. J Bacteriol. 2001;183:5213-22.

49. Maseda H, Sawada I, Saito K, Uchiyama H, Nakae T, Nomura N. Enhancement of the mexAB-oprM efflux pump expression by a quorum-sensing autoinducer and its cancellation by a regulator, MexT, of the mexEF-oprN efflux pump operon in Pseudomonas aeruginosa. Antimicrob Agents Chemother. 2004;48:1320-8.

50. Lamarche MG, Déziel E. MexEF-OprN efflux pump exports the Pseudomonas quinolone signal (PQS) precursor HHQ (4-hydroxy2-heptylquinoline). PLoS ONE. 2011;6:e24310.

51. Wade DS, Calfee MW, Rocha ER, Ling EA, Engstrom E, Coleman JP, et al. Regulation of Pseudomonas quinolone signal synthesis in Pseudomonas aeruginosa. J Bacteriol. 2005; 187:4372-80.

52. McGrath S, Wade DS, Pesci EC. Dueling quorum sensing systems in Pseudomonas aeruginosa control the production of the Pseudomonas quinolone signal (PQS). FEMS Microbiol Lett. 2004;230:27-34.

53. Déziel E, Lépine F, Milot S, He J, Mindrinos MN, Tompkins RG, et al. Analysis of Pseudomonas aeruginosa 4-hydroxy-2alkylquinolines (HAQs) reveals a role for 4-hydroxy-2- 
heptylquinoline in cell-to-cell communication. Proc Natl Acad Sci USA. 2004;101:1339-44.

54. Tian Z-X, Mac Aogain M, O'Connor HF, Fargier E, Mooij MJ, Adams C, et al. MexT modulates virulence determinants in Pseudomonas aeruginosa independent of the MexEF-OprN efflux pump. Microb Pathog. 2009;47:237-41.

55. Olivas AD, Shogan BD, Valuckaite V, Zaborin A, Belogortseva $\mathrm{N}$, Musch $\mathrm{M}$, et al. Intestinal tissues induce an SNP mutation in Pseudomonas aeruginosa that enhances its virulence: possible role in anastomotic leak. PloS ONE. 2012;7: e44326.

56. Köhler T, Michéa-Hamzehpour M, Henze U, Gotoh N, Kocjancic Curty L, Pechère $\mathrm{J}-\mathrm{C}$. Characterization of MexE-MexF-OprN, a positively regulated multidrug efflux system of Pseudomonas aeruginosa. Mol Microbiol. 1997;23:345-54.

57. Olivares J, Álvarez-Ortega C, Martinez JL. Metabolic compensation of fitness costs associated with overexpression of the multidrug efflux pump MexEF-OprN in Pseudomonas aeruginosa. Antimicrob Agents Chemother. 2014;58:3904-13.

58. Wang M, Schaefer AL, Dandekar AA, Greenberg EP. Quorum sensing and policing of Pseudomonas aeruginosa social cheaters. Proc Natl Acad Sci USA. 2015:112:2187-91.
59. Foster KR, Shaulsky G, Strassmann JE, Queller DC, Thompson CRL. Pleiotropy as a mechanism to stabilize cooperation. Nature . 2004:431:693-6.

60. Travisano M, Velicer GJ. Strategies of microbial cheater control. Trends Microbiol. 2004;12:72-8.

61. Mitri S, Foster KR. Pleiotropy and the low cost of individual traits promote cooperation. Evolution. 2016;70:488-94.

62. Minagawa S, Inami H, Kato T, Sawada S, Yasuki T, Miyairi S, et al. RND type efflux pump system MexAB-OprM of Pseudomonas aeruginosa selects bacterial languages, 3-oxo-acylhomoserine lactones, for cell-to-cell communication. BMC Microbiol. 2012;12:70.

63. Pearson JP, Van Delden C, Iglewski BH. Active efflux and diffusion are involved in transport of Pseudomonas aeruginosa cellto-cell signals. J Bacteriol. 1999;181:1203-10.

64. Evans K, Passador L, Srikumar R, Tsang E, Nezezon J, Poole K. Influence of the MexAB-OprM multidrug efflux system on quorum sensing in Pseudomonas aeruginosa. J Bacteriol. 1998;180:5443-7.

65. Chan YY, Chua KL. The Burkholderia pseudomallei BpeABOprB efflux pump: expression and impact on quorum sensing and virulence. J Bacteriol. 2005;187:4707-19. 